\title{
Local Anomaly Cancellation, $M$-Theory Orbifolds and Phase-Transitions
}

\author{
Michael Faux ${ }^{1}$, Dieter Lüst ${ }^{2}$ and Burt A. Ovrut ${ }^{3}$ \\ ${ }^{1}$ Departments of Mathematics and Physics \\ Columbia University \\ 2990 Broadway, New York, NY 10027 \\ ${ }^{2}$ Institut für Physik, Humboldt Universität \\ Invalidenstraße 110, 10115 Berlin, Germany \\ ${ }^{3}$ Department of Physics, University of Pennsylvania \\ Philadelphia, PA 19104-6396, USA
}

\begin{abstract}
In this paper we consider orbifold compactifications of M-theory on $S^{1} / \mathbf{Z}_{2} \times T^{4} / \mathbf{Z}_{2}$. We discuss solutions of the local anomaly matching conditions by twisted vector, tensor and hypermultiplets confined on the local orbifold six-planes. In addition we consider phase-transitions among different solutions which are mediated by Mtheory fivebranes which touch the local orbifold planes and are converted there to gauge instantons.
\end{abstract}




\section{Introduction}

$M$-theory harbors a broad spectrum of phenomena which can be systematically probed by analyzing anomalies in effective quantum field theories. In the case of orbifold compactifications of eleven-dimensional supergravity, a wide range of topological restrictions can be resolved, and the states localized on orbifold planes determined, by imposing factorization criteria on anomaly polynomials. The basic paradigm was espoused in [1, 2] by analyzing the $S^{1} / \mathbf{Z}_{2}$ compactification. This was successfully applied to the $T^{5} / \mathbf{Z}_{2}$ compactification in [3, 4]. In each of these cases, the orbifold planes comprise isolated, non-intersecting submanifolds. In more general situations, the orbifold planes can intersect, which gives rise to a number of novel features. In this paper, we describe local anomaly cancellation on $S^{1} / \mathbf{Z}_{2} \times T^{4} / \mathbf{Z}_{M}$ compactifications of $M$-theory, with $M=2,3,4$ or 6 . These correspond to special points in the moduli space of $S^{1} / \mathbf{Z}_{2} \times K 3$ compactifications. In such situations, the orbifold planes do intersect. These issues were first discussed in [5] and later in [6, 7]. Here, we greatly extend the analysis of local anomaly cancellation in orbifolds of this type, particularly emphasizing results pertaining to the simplest of these cases, corresponding to $M=2$.

In our previous work [5], we described the general features of $S^{1} / \mathbf{Z}_{2} \times T^{4} / \mathbf{Z}_{M} M$-theory orbifolds. In this paper, we expand those results, incorporating two subtle technical points which were not addressed in complete generality in our previous work. The first is an issue pertaining to the periodicity of the four-form $G$ that has recently been more thoroughly described in [8]. The second issue concerns the precise normalization of the $C G G$ term in the supergravity action. Each of these impinge numerically on our analyses, both in [5] and in this current paper, by changing the overall coefficient of the anomaly inflow due to the classical variation of the $C G G$ term. We treat this coefficient as a parameter, to be determined by consistency arguments, in a manner similar to the approach described in [9]. In order that $M$-fivebranes have unit magnetic charge, we choose a scale for the three-form potential $C$ such that, upon integration over dimensions transverse to a fivebrane, we obtain $\int d G=1$. This leaves only one coefficient in the basic Chern-Simons interactions of the effective field theory unconstrained by supersymmetry and by the requirement of fivebrane anomaly cancellation. However, this one parameter is uniquely fixed by the additional requirement of consistent orbifold compactifications and gives rise to the particular coefficient cited below for the $C G G$ inflow anomaly. As a result of these two described changes we now find a whole class of solutions of the local anomaly matching conditions, which in general consist of vector, hyper and tensor multiplets confined on the local orbifold six-planes and which also, for global consistency requirements, con- 
tain fivebranes, free to move in the 11-dimensional bulk (see also ref. [6] for discussion of solutions of the local anomaly equations).

This situation is interesting since it is the simplest scenario which involves fivebranemediated phase transitions in which the gauge group is nontrivially influenced by local tensor couplings. Specifically, if a fivebrane hits one of the local orbifold six-planes it will be described by a torsion free sheaf being equivalent to a small gauge instanton. Due to the presence of this instanton the original gauge group at the local orbifold plane will be broken to some subgroup, where also the number of tensor and hypermultiplets gets changed. In this way elements of a certain class of orbifold compactifications are related to each other by fivebrane-mediated phase transitions, where the associated magnetic charges at the six-dimensional orbifold planes are changing by one unit. In addition we also discuss the possibility of phase transitions with half-integer change of magnetic charge at the local six-planes. We suggest that these phase transitions are due to fivebranes which split at interconnecting ten-planes and are then transmuted to half-integrally charged gauge instantons.

The orbifold compactifications which we discuss in this paper are of interest for other reasons as well. For example, in a series of papers, the $S^{1} / \mathbf{Z}_{2}$ orbifold was compactified on smooth Calabi-Yau threefolds producing realistic "brane universe" theories of particle physics and cosmology [10]. In future work, we will explore both the formal and phenomenological aspects of different $M$-theory orbifold compactifications.

\section{$2 \quad S^{1} / \mathbf{Z}_{2} \times T^{4} / \mathbf{Z}_{M}$ Orbifolds}

The $S^{1} / \mathbf{Z}_{2} \times T^{4} / \mathbf{Z}_{M}$ orbifolds each involve a pair of ten-dimensional hyperplanes fixed under the $\mathbf{Z}_{2}$ projection, which we denote by $\alpha$, and a set of distinct seven-dimensional hyperplanes fixed under the $\mathbf{Z}_{M}$ projection, which we denote by $\beta$. Each of the $\beta$-planes transversally intersects each of the $\alpha$-planes once, at particular six-dimensional hyperplanes invariant under both $\alpha$ and $\beta$. Chiral anomalies are induced on the $\alpha$-planes and (separately) on the $\alpha \beta$-planes, due to localized chiral projections of fields. Cancellation of the ten-dimensional $\alpha$-plane anomalies is uniquely accomplished by an additional tendimensional $E_{8}$ Yang-Mills supermultiplet on each of the two $\alpha$-planes, as is well-known. In this paper, we concern ourselves with the six-dimensional $\alpha \beta$-plane anomalies.

Although some of our discussion will be more general, it is helpful to have a specific orbifold in mind to help visualize the basic geometric setting. Our prototype is the simplest of the orbifolds described above, namely, the $S^{1} / \mathbf{Z}_{2} \times T^{4} / \mathbf{Z}_{2}$ orbifold. In this case, spacetime has topology $\mathbf{R}^{6} \times T^{5}$ and each of the five compact coordinates takes 


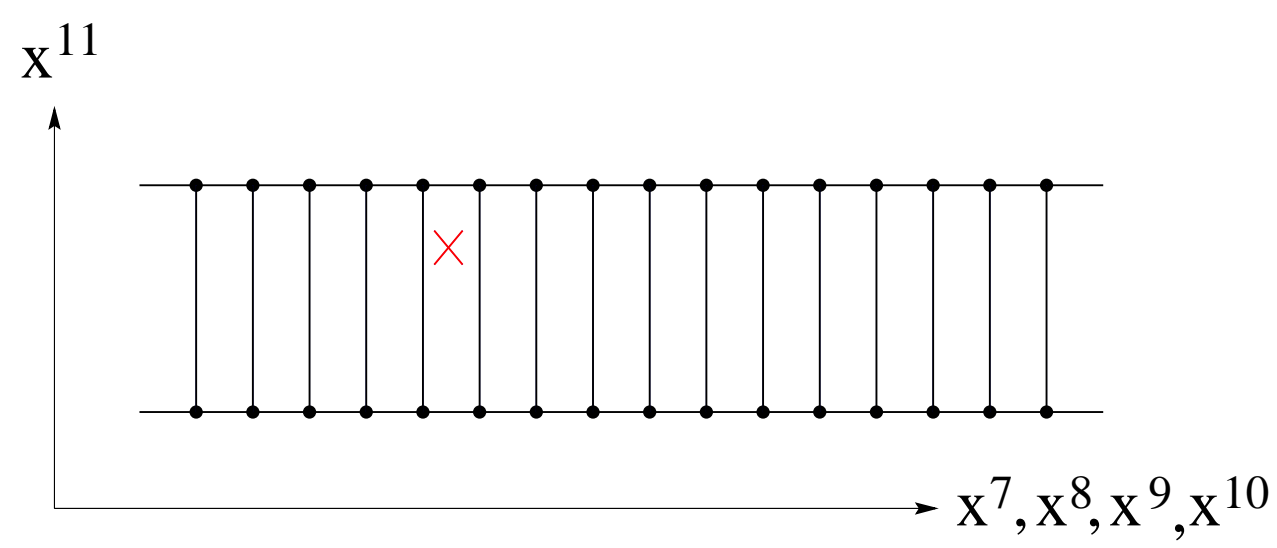

Figure 1: The global structure of orbifold planes in the $S^{1} / \mathbf{Z}_{2} \times T^{4} / \mathbf{Z}_{2}$ orbifold. Horizontal lines represent the two ten-dimensional $\alpha$-planes, while vertical lines represent the sixteen seven-dimensional $\beta$-planes. The thirty-two six-dimensional $\alpha \beta$-planes are represented by the solid dots and coincide with the intersection of the $\alpha$-planes and the $\beta$-planes. The $X$ in the figure indicates a "wandering" fivebrane.

values on the interval $[-\pi, \pi]$ with the endpoints identified. The nontrivial projections are $\alpha:\left(x^{\mu}, x^{i}, x^{11}\right) \longrightarrow\left(x^{\mu}, x^{i},-x^{11}\right)$ and $\beta:\left(x^{\mu}, x^{i}, x^{11}\right) \rightarrow\left(x^{\mu},-x^{i}, x^{11}\right)$, where $x^{\mu}$ parameterizes the six noncompact dimensions, while $x^{i}$ and $x^{11}$ parameterize the $T^{4}$ and $S^{1}$ factors respectively. The element $\alpha$ leaves invariant the two ten-planes defined by $x^{11}=0$ and $x^{11}=\pi$, while $\beta$ leaves invariant the sixteen seven-planes defined when the four coordinates $x^{i}$ individually assume the values 0 or $\pi$. Finally, $\alpha \beta$ leaves invariant the thirty-two six-planes defined when all five compact coordinates individually assume the values 0 or $\pi$. The $\alpha \beta$ six-planes coincide with intersections of the $\alpha$ ten-planes with the $\beta$ seven-planes. The global structure is depicted in Figure 1.

There are several magnetic and electric sources for $G$ necessary to resolve chiral anomalies in these orbifolds. The basic Chern-Simons terms include the $C G G$ interaction and also the higher-derivative $G X_{7}$ interaction, where $X_{8} \equiv d X_{7}$ is the eight-form describing the worldvolume anomaly generated by the fivebrane zero modes. The worldvolume anomaly is cancelled by inflow mediated by the $G X_{7}$ interaction, provided the fivebrane acts as a magnetic source for $G$, in the sense described above. Next, each of the two $\alpha$-planes supports a ten-dimensional $E_{8}$ super-Yang-Mills multiplet. They also provide magnetic sources for $G$ due to the presence of terms $\delta^{(1)} I_{4}$ in the $d G$ bianchi identity, where $\delta^{(1)}$ is a one-form brane-current localized on the $\alpha$-plane and $I_{4}$ is a four-form polynomial involving the Lorentz-valued curvature $R$ and the local $E_{8}$ field-strength $F$.

The seven-dimensional $\beta$-planes provide electric sources for $G$ via Chern-Simons interactions $\int \delta^{(4)} G Y_{3}^{0}$, where $\delta^{(4)}$ is a four-form brane-current localized on the seven-plane 
and $Y_{4}=d Y_{3}^{0}$ is a gauge-invariant four-form polynomial. This polynomial involves the curvature $R$ and also a field strength $\mathcal{F}$ associated with additional adjoint super-gauge fields localized on the seven-plane (with the gauge group determined by anomaly cancellation in a manner which we will describe). This coupling gives rise to an "I-brane" effect via interplay with the ten-dimensional magnetic source (involving $I_{4}$ ). This contributes additional inflow localized on the six-dimensional intersection of the ten-dimensional $\alpha$-plane and the seven-dimensional $\beta$-plane $\mathrm{U}$.

The magnetic and electric sources described so far are encapsulated by the following three polynomials

$$
\begin{aligned}
X_{8}(R) & =\frac{1}{(2 \pi)^{3} 4 !}\left(\frac{1}{8} \operatorname{tr} R^{4}-\frac{1}{32}\left(\operatorname{tr} R^{2}\right)^{2}\right) \\
I_{4}(R, F) & =\frac{1}{16 \pi^{2}}\left(-\frac{1}{2} \operatorname{tr} R^{2}+\operatorname{tr} F^{2}\right) \\
Y_{4}(R, \mathcal{F}) & =\frac{1}{4 \pi}\left(-\frac{1}{32} \eta \operatorname{tr} R^{2}+\rho \operatorname{tr} \mathcal{F}^{2}\right) .
\end{aligned}
$$

The precise forms of $X_{8}$ and $I_{4}$ are fixed by fivebrane consistency and ten-dimensional anomaly cancellation, respectively. The polynomial $Y_{4}$ is parameterized by two rational coefficients $\eta$ and $\rho$. These are determined by further requirements described below.

Finally, the six-dimensional $\alpha \beta$-planes carry a magnetic charge. This appears in the $d G$ Bianchi identity as a term $g \delta^{(5)}$, where $\delta^{(5)}$ is a five-form brane-current localized on the $\alpha \beta$-plane and $g$ is a rational magnetic charge subject to a quantization condition. For the case $M=2$, the magnetic charge $g$ should be quarter-integer, as explained in [5]. The Bianchi identity for the four-form field strength $G$ is, therefore, given by

$$
d G=\sum_{i=1}^{2} I_{4(i)} \delta_{M_{i}^{10}}^{(1)}+\sum_{i=1}^{2 f} g_{i} \delta_{M_{i}^{6}}^{(5)}+\sum_{i=1}^{N_{5}} \delta_{W_{i}^{6}}^{(5)}
$$

where we have included all of the magnetic sources described above. The manifold $M_{i}^{10}$ is the $i$ th $\alpha$-plane, while $M_{i}^{6}$ is the $i$ th $\alpha \beta$-plane and $W_{i}^{6}$ is the worldvolume of the $i$ th fivebrane [2. In most of this paper, our expressions apply to a particular $\alpha \beta$-plane. Hence, the label $i$ is implicit but omitted.

The projection $\beta$ can independently break the ten-dimensional $E_{8}$ gauge groups on the $\alpha \beta$-planes to maximal subgroups. Since a ten-dimensional vector supermultiplet decomposes into one six-dimensional $N=1$ vector and one six-dimensional hypermultiplet,

\footnotetext{
${ }^{1}$ See Section 5 of $[5$ ] for a description of this effect

${ }^{2}$ The pervasive use of the label $i$ is merely convenient, and does not imply any specific correlation between these manifolds.
} 
the breaking pattern will be characterized by an integer number $V_{B}$ of vector multiplets and another integer number $H_{B}$ of hypermultiplets, each transforming according to some representation $\mathcal{R}$ of the residual maximal subgroup of $E_{8}$. The projection $\beta$ necessarily removes half of the $E_{8}$ degrees of freedom. But the identity of which half depends on how $\beta$ acts on the $E_{8}$ root lattice.

Chiral projection of the supergravity fields results in further contributions to the local $\alpha \beta$ anomalies. These derive from "untwisted" fields comprising one universal $N=1$ tensor multiplet and some number $h$ of hypermultiplets. The value of $h$ depends on which $Z_{M}$ orbifold is being considered. For the cases $M=2$ and $M=3$, we have $h=4$ and $h=2$ respectively. Furthermore, since the local anomaly due to the supergravity and residual $E_{8}$ fields arises from the coupling of fields which are not themselves localized on the $\alpha \beta$-planes, it involve fractions which reflect the multiplicies of the fixed planes. We parameterize this by another integer $f$ corresponding to the number of $\beta$-planes associated with the orbifold in question. For the cases $M=2$ and $M=3$, we have $f=16$ and $f=9$ respectively. These correspond to the respective sixteen and nine fixed-planes in the $Z_{2}$ and $Z_{3}$ orbifold limits of the $K 3$ manifold 3 .

Finally, we allow for as yet unspecified $N=1$ supermatter localized on each $\alpha \beta$-plane. We call this matter "twisted", since it is analogous to twisted sector matter in superstring orbifolds. This matter assembles into $n_{T}$ tensor multiplets, $n_{V}$ vector multiplets and $n_{H}$ hypermultiplets, and involves an as yet undetermined "twisted" gauge group $\tilde{\mathcal{G}}$. The vector multiplets transform in the adjoint representation, while the hypermultiplets transform in an unspecified representation $\tilde{\mathcal{R}}$.

We focus on a particular $\alpha \beta$-plane, and assemble each of the contributions to the six-dimensional anomaly localized on this plane. There are three classical (inflow) contributions, described by the polynomials

$$
\begin{aligned}
I_{8}(C G G) & =-\pi g I_{4}(R, F)^{2} \\
I_{8}\left(G X_{7}\right) & =-g X_{8}(R) \\
I_{8}(I B) & =-I_{4}(R, F) \wedge Y_{4}(R, \mathcal{F}) .
\end{aligned}
$$

The first two arise from the variation of the $C G G$ and $G X_{7}$ terms. The third arises from the variation of $\int \delta^{(4)} G Y_{3}^{0}$ and describes the "I-brane" anomaly th. There are also three

\footnotetext{
${ }^{3}$ The $Z_{4}$ and $Z_{6}$ orbifolds involve additional subtlety which we will not discuss in this paper.

${ }^{4}$ The necessity for the "I-brane" contribution in an orbifold context was first recognized in [5], and was inspired by an analogous effect on intersecting D-branes, introduced in [12].
} 
quantum contributions

$$
\begin{aligned}
I_{8}(S G)= & \frac{1}{2 f}\left(I_{G R A V}^{(3 / 2)}(R)-(1+h) I_{G R A V}^{(1 / 2)}(R)\right) \\
I_{8}(\mathcal{G})= & \frac{1}{f}\left(\left(V_{B}-H_{B}\right) I_{G R A V}^{(1 / 2)}(R)+I_{M I X E D}^{(1 / 2)}(R, F)_{\mathcal{R}}+I_{G A U G E}^{(1 / 2)}(F)_{\mathcal{R}}\right) \\
I_{8}(\tilde{\mathcal{G}})= & \left(n_{V}-n_{H}-n_{T}\right) I_{G R A V}^{(1 / 2)}(R)-n_{T} I_{G R A V}^{(3-\text { form })}(R) \\
& +I_{M I X E D}^{(1 / 2)}(R, \mathcal{F})_{\tilde{\mathcal{R}}}+I_{G A U G E}^{(1 / 2)}(\mathcal{F})_{\tilde{\mathcal{R}}} .
\end{aligned}
$$

The factors $I_{\mathrm{GRAV}}, I_{M I X E D}$ and $I_{\text {GAUGE }}$ which appear in the quantum anomalies describe one-loop gravitational, mixed and pure-gauge anomalies. They are attributable to the type of chiral fields with spin indicated by the superscripts. These are determined by index theorems and are listed explicitly in appendix $\mathrm{C}$ of [5]. The anomaly $I_{8}(\mathcal{G})$ describes the local anomaly involving whatever subgroup $\mathcal{G} \subset E_{8}$ is left unbroken by $\beta$ on the relevant $\alpha \beta$-plane. The subscript $\mathcal{R}$ indicates the representation content of the $E_{8}$ residual subgroup. Hence, the traces over the gauge factors in $I_{M I X E D}^{(1 / 2)}(R, F)_{\mathcal{R}}$ and $I_{G A U G E}^{(1 / 2)}(F)_{\mathcal{R}}$ are traces over $\mathcal{R}$. Similar comments apply to the $\alpha \beta$ twisted sector with gauge group $\tilde{\mathcal{G}}$, where the representation content is indicated by the subscript $\tilde{\mathcal{R}}$. f

There are numerous unspecified parameters involved in the six contributions in (3) and (舟). To begin with, there are ten integers characterizing the global geometry of the orbifold, the local $E_{8}$ breaking pattern and multiplicities in the twisted and untwisted spectra. For instance, the orbifold geometry is partially encoded in the number $f$ of $\beta$-fixed planes and the number $h$ of universal untwisted hypermultiplets. The magnetic charge $g$ is an integer times a basic quantization unit (which also reflects the orbifold geometry). The two parameters $\eta$ and $\rho$ describe electrical charges of the seven-planes, as defined in equation (11). The $E_{8}$ breaking pattern is encoded in the multiplicities $V_{B}$ and $H_{B}$. Finally, the local twisted spectrum is encoded in the multiplicities $n_{V}, n_{H}$ and $n_{T}$.

Furthermore, there are six sets of rational parameters which characterize the representation content of the matter fields. These are given by "representation indices", which allow one to relate traces over a given representation to traces over the fundamental representation. The relevant representation indices are denoted $\mathcal{I}_{2}(\mathcal{R}), \mathcal{I}_{2,2}(\mathcal{R})$ and $\mathcal{I}_{4}(\mathcal{R})$,

\footnotetext{
${ }^{5}$ The technical aspects involved in determining (3) and (4) are described in [5]. The determination of $I_{8}(C G G)$ is more subtle than indicated in that paper, however, for reasons mentioned previously. See [8] for a more thorough description of this one term. Note that the twisted gauge group $\tilde{\mathcal{G}}$ can include factors which coincide with some factors in the $E_{8}$ residual group $\mathcal{G}$.
} 


\begin{tabular}{cc|ccc}
$\mathcal{G}$ & $\mathcal{R}$ & $I_{4}(\mathcal{R})$ & $I_{2,2}(\mathcal{R})$ & $I_{2}(\mathcal{R})$ \\
\hline$E_{8}$ & $\mathbf{2 4 8}$ & 0 & 9 & 30 \\
$E_{7}$ & $\mathbf{1 3 3}$ & 0 & $1 / 6$ & 3 \\
& $\mathbf{5 6}$ & 0 & $1 / 24$ & 1 \\
$S O(16)$ & $\mathbf{1 2 0}$ & 8 & 3 & 14 \\
& $\mathbf{1 2 8}$ & -8 & 6 & 16 \\
& $\mathbf{1 6}$ & 1 & 0 & 1 \\
$S U(2)$ & $\mathbf{3}$ & 0 & 8 & 4 \\
& $\mathbf{2}$ & 0 & $1 / 2$ & 1
\end{tabular}

Table 1: Some useful representation indices.

and are defined by

$$
\begin{aligned}
& \operatorname{tr}_{\mathcal{R}} F^{2}=\mathcal{I}_{2}(\mathcal{R}) \operatorname{tr} F^{2} \\
& \operatorname{tr}_{\mathcal{R}} F^{4}=\mathcal{I}_{2,2}(\mathcal{R})\left(\operatorname{tr} F^{2}\right)^{2}+\mathcal{I}_{4}(\mathcal{R}) \operatorname{tr} F^{4},
\end{aligned}
$$

where all traces on the right-hand side are over the fundamental representation. Some useful representation indices are listed in Table 1 ?.

Remarkably, each and every one of the above parameters can be resolved, in a sense to be made clear, by imposing necessary factorization properties on the net anomaly obtained by summing the six contributions listed in (31) and (41). That result describes the total local anomaly on a particular $\alpha \beta$-plane due to the sources which we have described so far. Since the total local anomaly must vanish, there are two possibilities. The first is that the net anomaly vanishes identically. The remaining possibility is that this net anomaly is non-zero, but is cancelled by a contribution arising from special couplings of local tensor fields which, thereby, provide a local Green-Schwarz mechanism.

Local tensor fields reside in twisted $N=1$ tensor multiplets on the $\alpha \beta$-plane. The two-form fields in such a multiplet are anti-self-dual, in the sense that the associated gauge-invariant three-form field-strength satisfies $H=-* H$. As a result of this, a special Chern-Simons interaction $\int \delta^{(5)} H Z_{3}^{0}$, where $\delta^{(5)}$ is the five-form brane-current with

\footnotetext{
${ }^{6}$ The evaluation of the representation indices for arbitrary representations of the classical Lie groups is a complicated problem. See [11] for a discussion of this issue.
} 
support on the $\alpha \beta$-plane and $Z_{4}=d Z_{3}^{0}$ is a gauge-invariant four-form polynomial, will lead to two consequences. First, due to the anti-self-duality, the electric coupling described by the Chern-Simons interaction implies a magnetic coupling described by the Bianchi identity $d H=Z_{4}$. The magnetic coupling involves the same polynomial as the electric coupling because anti-self-duality is equivalent to an electric-magnetic duality Secondly, the Chern-Simons coupling generates an inflow anomaly described by $I_{8}(G S)=Z_{4} \wedge Z_{4}$, where $G S$ designates Green-Schwarz. Note that this polynomial is a perfect square due to the duality. This is, in turn, a consequence of $N=1$ supersymmetry. Thus, the net anomaly can be cancelled by specialized local tensor dynamics, provided that the net anomaly reduces to a sum of perfect squares with one term for each available tensor field.

\section{Local Anomaly Cancellation}

As a result of the above discussion, a program for analyzing the local anomaly on a given $\alpha \beta$-plane becomes apparent. First, we assemble the net anomaly by summing the six terms in (3) and (44). We will call this result $I_{8}$. Then we sequence through the possibilities $n_{T}=0,1,2, \ldots$, in each case imposing that $I_{8}$ satisfies the appropriate factorization requirement. For the case $n_{T}=0$, since there are no local tensors, we require $I_{8}=0$. For the case $n_{T}=1$, we impose that $I_{8}$ is proportional to a complete square, that is, $I_{8} \propto\left(Z_{4}\right)^{2}$. (In this second case, we simultaneously determine the form of the electric and magnetic couplings of the local tensor.) For the case $n_{T}=2$, we require that $I_{8}$ is the sum of two perfect squares. And so forth. These factorization requirements prove to be marvelously restrictive. For each choice of $n_{T}$, there results unambiguous values for each and every one of the previously unspecified geometric and topological parameters, including the values of the magnetic charge, electric charges, and the identity of the gauge groups and representation content of the twisted sectors.

Since $M$-fivebranes carry unit magnetic charge, as well as the zero mode fields described previously, we infer that a fivebrane moving onto (or off of) a given $\alpha \beta$-plane deposits (or removes) charge and twisted modes in the process of doing so. Since the fivebrane carries one $N=1$ tensor multiplet and one hypermultiplet, we expect that the solutions to our factorization constraints will assemble into hierarchies linked by incrementing the magnetic charge and the local tensor and hyper multiplicities as $g \rightarrow g+1, n_{T} \rightarrow n_{T}+1$ and $n_{H} \rightarrow n_{H}+1$. This is precisely what we find. Mathematically, this can be understood as follows. A single fivebrane touching an orbifold fixed plane is described by a singular

\footnotetext{
${ }^{7}$ This is easy to see by taking the exterior derivative of the Euler-Lagrange equation $* H=-Z_{3}$, and then replacing $* H$ with $-H$.
} 
object called a "torsion free sheaf" [13]. This sheaf carries one extra unit of magnetic charge and has one tensor multiplet and one hypermultiplet as zero modes, identical to the analogous fivebrane data. This accounts for the increase in each of these quantities by unity when the fivebrane is moved to a fixed plane.

At least in some cases, the torsion free sheaf can be shown to be the singular "small instanton" limit of a smooth gauge instanton [14]. Smoothing out the sheaf into an instanton represents a true phase transition, where the fivebrane data disappears and is replaced by a vector bundle [10]. In this process, the unit magnetic charge of the sheaf is replaced by a unit increase in the second Chern number of the vector bundle. The zero modes of the vector bundle are, in general, quite different from those of the torsion free sheaf. Most importantly, the appearance of a smooth, non-trivial vector bundle signals the breakdown of the original twisted sector gauge group to a smaller group $\mathcal{G} \subset E_{8}$. Therefore, after this phase transition, we expect to have a smaller twisted sector gauge group with identical topological charge but different numbers of tensor and hypermultiplets. Be that as it may, this theory remains anomaly free. As we will see, locally anomaly free orbifold planes do exist that could be related to each other through small instanton phase transitions. Thus, factorization of the local anomaly polynomial yields an extra bonus.

In addition to the torsion free sheaf transitions described earlier, we expect another interesting grouping of our factorization solutions. In this second grouping, we expect small instanton transitions between sets of solutions with identical local magnetic charge, but with different numbers of zero modes and different local gauge groups.

For clarity, let us recapitulate the fivebrane transitions discussed in the previous two paragraphs. If a fivebrane moves to a particular $\alpha \beta$-plane, it should increment the local magnetic charge by one, and add one local tensor and one local hypermultiplet to the associated twisted spectrum. We would then attribute one unit of the total local magnetic charge to the latent magnetic charge of the fivebrane, now interpreted as a torsion-free sheaf. The associated tensor would be available to help mediate anomaly cancellation through a local Green-Schwarz mechanism. Now, assume this configuration is a small instanton and can be deformed to a smooth vector bundle. Then, since the instanton does not have a tensorial zero mode, there would be one less tensor available to mediate the anomaly cancellation. The anomaly polynomial should, therefore, reconfigure so as to ensure continued anomaly cancellation, but with a modified factorization criterion. Thus, by classifying independent solutions to the factorization requirements into sets involving identical values of $g$ but different numbers of tensor fields, one can infer such nontrivial phase transitions. 


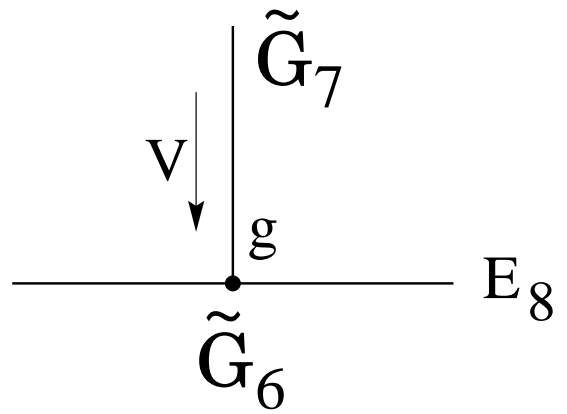

Figure 2: The local geometry near a particular $\alpha \beta$-plane, showing some of the data to be resolved by anomaly factorization.

We begin our analysis by considering a particular $\alpha \beta$ plane, corresponding to one of the solid dots in Figure 1, representing the unique six-dimensional intersection of a particular ten-dimensional $\alpha$-plane and a particular seven-dimensional $\beta$-plane. To be more concrete, we focus on one of the intersection points on the lower of the two $\alpha$ planes in Figure 1, so that the local geometry is depicted as in Figure 2. In Figure 2, the horizontal line represents the $\alpha$-plane, the vertical line represents the $\beta$-plane and, finally, the point of intersection represents the $\alpha \beta$-plane. The intersection supports the local anomaly in which we are interested and has magnetic charge $g$. The $\alpha$-plane supports $E_{8}$ super Yang-Mills fields, as described above. This $E_{8}$ group is, in general, broken on the $\alpha \beta$-plane to some subgroup depending of the action of $\beta$ on the $E_{8}$ root lattice. In the vertex-diagrams below, we indicate, to the right of the horizontal lines, the subgroup of $E_{8}$ left unbroken at the intersection. Thus, Figure 2 indicates a scenario in which the full $E_{8}$ is left unbroken. This figure also indicates the presence of additional gauge structure with group $\tilde{\mathcal{G}}_{7}$ localized on the $\beta$-plane and further gauge structure on the $\alpha \beta$-plane with group $\tilde{\mathcal{G}}_{6}$. These correspond to additional seven-dimensional and six-dimensional fields, respectively.

The only multiplet in seven dimensions is a vector multiplet, which transforms in the adjoint of $\tilde{\mathcal{G}}_{7}$. This decomposes into one six-dimensional $N=1$ vector multiplet and one six-dimensional hypermultiplet 5 . An important fact is that the seven-dimensional fields are chirally projected by $\alpha$ onto the embedded six-dimensional $\alpha \beta$-plane. It, thereby, contributes to the local $\alpha \beta$ anomaly.

If $\tilde{\mathcal{G}}_{7}$ does not coincide with some factor of the broken subgroup of $E_{8}$, then sixdimensional gauge invariance dictates that the hypermultiplet is the part projected out by $\alpha$. Thus, the $\tilde{\mathcal{G}}_{7}$ gauge fields remain at the intersection to enforce local $\tilde{\mathcal{G}}_{7}$ invariance.

\footnotetext{
${ }^{8}$ As a point of interest, this is the same decomposition enjoyed by a ten-dimensional vector multiplet.
} 
The $\tilde{\mathcal{G}}_{7}$ adjoint gauginos fill out a six-dimensional $N=1$ vector multiplet, which is indicated by the $V$ next to the downward arrow in Figure 2. This tells us that the "vector" part survives the $\alpha$ projection as we move down the vertical line in that diagram and land on the intersection point. Since the $N=1$ vector multiplet is chiral, the $\tilde{\mathcal{G}}_{7}$ gauginos will contribute to the $\alpha \beta$ anomaly. However, since this anomaly results from the coupling of fields not localized on the $\alpha \beta$-plane, this anomaly must be divided by two (since there are two $\alpha \beta$-planes embedded within a given $\beta$-plane) compared to a similar anomaly due to six-dimensional $\tilde{\mathcal{G}}_{7}$ adjoint gauginos.

If $\tilde{\mathcal{G}}_{7}$ coincides with a factor in the unbroken subgroup of $E_{8}$, then the $\tilde{\mathcal{G}}_{7}$ gauge fields on the $\alpha \beta$-plane may be supplied by the ten-dimensional gauge fields which survive the $\beta$ projection. Consequently, the projection $\alpha$ should remove the "vector" part of the seven-dimensional adjoint matter, so that the other part, corresponding to an adjoint hypermultiplet, survives the $\alpha$ projection. We indicate this alternate situation by an $H$ next to the downward arrow in the corresponding diagram. In this case, the surviving hyperinos would contribute to the local anomaly. This anomaly would include a division by two compared to a similar anomaly due to six-dimensional $\tilde{\mathcal{G}}_{7}$ adjoint hyperinos, for reasons identical to those described in the preceeding paragraph 9 .

A third possibility is that the group $\tilde{\mathcal{G}}_{7}$ is broken, by $\beta$, to some maximal subgroup $\mathcal{H} \subset \tilde{\mathcal{G}}_{7}$ on the $\alpha \beta$-plane. In this case, the seven-dimensional fields would decompose into various six-dimensional fields transforming according to representations determined by the appropriate branching rule. These would include fields transforming in the adjoint of $\mathcal{H}$ and other fields transforming in other representations of $\mathcal{H}$. The vector part of the adjoint fields would survive the $\beta$ projection while the hypermultiplet part of the remaining fields would survive. We indicate this hybrid situation by replacing the $V$ in Figure 2 with the relevant subgroup $\mathcal{H} \subset \tilde{\mathcal{G}}_{7}$ which survives the projection.

In resolving the factorization criteria necessary to explain local anomaly cancellation on a given $\alpha \beta$-plane, one requires factors of two which can only be explained by sevendimensional matter in the manner we have just described. Thus, the identity of sevendimensional matter is indicated by anomaly cancellation on an embedded sub-plane. This is interesting because the seven-plane itself cannot support a local anomaly since it is odddimensional. (The situation is analogous to the fact that eleven-dimensional supergravity is needed by the $E_{8}$ super-gauge multiplets on the two $\alpha$-planes to render those ten-planes anomaly-free.)

There is one more subtle factor of one-half which needs explanation. This relates to

\footnotetext{
${ }^{9}$ The possibility of alternatively projecting out the vector or hypermultiplet parts of the sevendimensional fields on the six-dimensional planes was also mentioned in [6].
} 
hypermultiplets in the six-dimensional twisted sector. It is possible for $r$ hypermultiplets to transform according to a $2 r$ dimensional representation $\tilde{\mathcal{R}}$ of the gauge group $\tilde{\mathcal{G}}$, provided the representation is "pseudoreal" in a sense to be clarified. In this case, the $4 r$ scalar fields assemble into $r$ quaternions represented as $\phi_{i}^{\alpha}$, where $i=1,2$ is an index which spans the $\mathbf{2}$ represention of an $S p(1)$ automorphism of the supersymmetry algebra and $\alpha=1, \ldots, 2 r$ spans $\tilde{\mathcal{R}}$. The group $\tilde{\mathcal{G}}$ acts as $\delta \phi_{i}^{\alpha}=\theta^{a}\left(T_{a}\right)^{\alpha}{ }_{\beta}$, where $\left(T_{a}\right)^{\alpha}{ }_{\beta}$ are antihermitian generators. There exists a real invariant tensor $\rho^{\alpha \beta}=\rho_{\alpha \beta}$ which, by suitable field redefinition, can be put into block-diagonal form $\rho=\operatorname{diag}\left(i \sigma_{2} \cdots i \sigma_{2}\right)$, where $\sigma_{2}$ is the second Pauli matrix. The representation is pseudoreal if $\left(T_{a}^{*}\right)=-\rho T_{a} \rho$. See [15, 16] for a more comprehensive discussion of hypermultiplets. In this case, we refer more properly to $2 r$ half-hypermultiplets, since the number of hypermultiplets is half the dimensionality of the representation. Each such half-hypermultiplet then contributes one-half of the anomaly which we would normally attribute to $2 r$ antichiral spinors transforming in $\tilde{\mathcal{R}}$ via naive application of index theorems.

Next, we should explain how to algebraically characterize the possible branching patterns describing the projection of the two $E_{8}$ factors by $\beta$. The simplest possibility is the one indicated in Figure 2, where the relevant $E_{8}$ factor remains unbroken. In terms of six-dimensional $N=1$ multiplets, the ten-dimensional $E_{8}$ vector multiplet decomposes into one vector multiplet and one hypermultiplet. In this case, it is the hypermultiplet components which are projected out by $\beta$ on the $\alpha \beta$-plane. This leaves us with the gauge fields necessary to enforce local $E_{8}$ invariance on the $\alpha \beta$-plane. This also fixes two of our parameters, $\left(V_{B}, H_{B}\right)=(248,0)$. In this case, all $E_{8}$ traces which appear in both the inflow anomalies (3) and the quantum anomalies (10) are each taken at face value. Thus, using the representation indices in Table 1 , we can use the results $\operatorname{Trace}_{\mathbf{2 4 8}} F^{2} \equiv 30 \operatorname{tr} F^{2}$ and $\operatorname{Trace}_{\mathbf{2 4 8}} F^{4}=9\left(\operatorname{tr} F^{2}\right)^{2}$ to express the traces which appear in the quantum anomalies (4) in terms of the fundamental (tr) traces

More generally, the $E_{8}$ factors will be broken by $\beta$, on the $\alpha \beta$-planes, to some maximal subgroup with a branching pattern which can be found from the tables in [17]. In this case, when we determine our anomaly polynomial $I_{8}$ by adding up the six contributions in (3) and (田), we replace the various $E_{8}$ traces by traces over the relevant representations of the residual subgroup. We then relate these to traces over fundamental representations of the factors in this subgroup by using representation indices, such as those listed in Table 1. However, there is a subtle difference between the inflow contributions (3) and the quantum contributions (目) which should be properly accounted for, and which we now describe.

\footnotetext{
${ }^{10}$ Note that the traces which appear in the inflow anomaly (3), through the implicit dependence of (1), are fundamental traces to begin with.
} 
Since the inflow anomalies are classical expressions, we can apply the group theoretic reduction directly on the traces which appear in (3). However, in the general case, the six-dimensional quantum anomalies derive from both chiral and antichiral fields. The chiral fields, which satisfy $\Gamma_{7} \psi=\psi$, appear in the six-dimensional $N=1$ vector multiplets. The antichiral fields, which satisfy $\Gamma_{7} \psi=-\psi$, occur in hypermultiplets. Since chiral and antichiral fields contribute one-loop anomalies with opposite sign, there is an extra minus sign associated with all quantum anomalies arising from hypermultiplet couplings. We illustrate this with two explicit examples.

As a first example, we choose the breaking pattern $E_{8} \rightarrow E_{7} \times S U(2)$. In this case, we have the branching rule $248=(\mathbf{1 3 3}, \mathbf{1}) \oplus(\mathbf{1}, \mathbf{3}) \oplus(\mathbf{5 6}, \mathbf{2})$. We determine that the surviving six-dimensional fields comprise $133 N=1$ vector multiplets transforming as the adjoint of $E_{7}$, another three vector multiplets transforming as the adjoint of $S U(2)$ and 112 hypermultiplets transforming as a bifundamental representation. Thus, $\left(V_{B}, H_{B}\right)=$ $(136,112)$. In this case, we reduce the $E_{8}$ traces which occur in the inflow anomaly as follows

$$
\begin{aligned}
\operatorname{tr} F^{2} & =\frac{1}{30} \operatorname{Tr}_{\mathbf{2 4 8}} F^{2} \\
& =\frac{1}{30}\left(\operatorname{Tr}_{\mathbf{1 3 3}} F_{a}^{2}+2 \operatorname{tr}_{\mathbf{5 6}} F_{a}^{2}+\operatorname{Tr}_{\mathbf{3}} F_{b}^{2}+56 \operatorname{tr}_{\mathbf{2}} F_{b}^{2}\right) \\
& =\frac{1}{6} \operatorname{tr} F_{a}^{2}+2 \operatorname{tr} F_{b}^{2},
\end{aligned}
$$

where we have used the indices in Table 1. In (6), the subscripts $a$ and $b$ denote $E_{7}$ and $S U(2)$ respectively. In the final line, we have dropped the labels from the fundamental 56 and 2 traces. Thus, to describe the inflow anomaly in the case where the $E_{8}$ factor is broken by $\beta$ to $E_{7} \times S U(2)$, we substitute the identity (6) for the $\operatorname{tr} F^{2}$ in the inflow anomaly (3). In the quantum anomaly, on the other hand, hypermultiplets and vector multiplets contribute with opposite signs. As a result, when computing the local one-loop anomaly in the case where $E_{8} \rightarrow E_{7} \times S U(2)$, we we should replace the factor trace $F^{2}$ which appears in $I_{\mathrm{MIXED}}^{(1 / 2)}$ using the following $\square$

$$
\begin{aligned}
\operatorname{trace} F^{2} & =\operatorname{Tr}_{\mathbf{2 4 8}} F^{2} \\
& =\operatorname{Tr}_{\mathbf{1 3 3}} F_{a}^{2}-2 \operatorname{tr}_{\mathbf{5 6}} F_{a}^{2}+\operatorname{Tr}_{\mathbf{3}} F_{b}^{2}-56 \operatorname{tr}_{\mathbf{2}} F_{b}^{2} \\
& =\operatorname{tr} F_{a}^{2}-52 \operatorname{tr} F_{b}^{2} .
\end{aligned}
$$

This derivation differs from (6) by the minus signs on terms relating to hypermultiplet

\footnotetext{
${ }^{11}$ See equation (C.2) of [5] for the explicit polynomial corresponding to $I_{M I X E D}^{(1 / 2)}$, as well as all of the other quantum anomaly polynomials referred to in this paper.
} 
couplings. As described above, these minus signs reflect the antichirality of hyperinos. Similar comments apply to the term trace $F^{4}$ which appears in $I_{\text {GAUGE }}^{(1 / 2)}$ In total, to describe the case $E_{8} \rightarrow E_{7} \times S U(2)$ we should make the following replacements

$$
\begin{aligned}
\operatorname{tr} F^{2} & =\frac{1}{6} \operatorname{tr} F_{a}^{2}+2 \operatorname{tr} F_{b}^{2} \\
\operatorname{trace} F^{2} & =\operatorname{tr} F_{a}^{2}-52 \operatorname{tr} F_{b}^{2} \\
\operatorname{trace} F^{4} & =\frac{1}{12}\left(\operatorname{tr} F_{a}\right)^{2}-20\left(\operatorname{tr} F_{b}\right)^{2}-6 \operatorname{tr} F_{a}^{2} \wedge \operatorname{tr} F_{b}^{2} .
\end{aligned}
$$

The first of these should be substituted in the classical (inflow) anomaly, while the second two should be substituted in the quantum anomaly. Note our mnemonic that traces which appear in the quantum anomaly are designated "trace", whereas classical traces are abbreviated "tr".

As a second example, we choose the breaking pattern $E_{8} \rightarrow S O(16)$. In this case, we have the branching rule $\mathbf{2 4 8} \rightarrow \mathbf{1 2 0} \oplus \mathbf{1 2 8}$. We determine that the surviving sixdimensional fields comprise $120 N=1$ vector multiplets transforming as the adjoint and 128 hypermultiplets transforming as the spinor of $S O(16)$. Thus, $\left(V_{B}, H_{B}\right)=(120,128)$. In this case, we reduce the $E_{8}$ traces which occur in the inflow anomaly and in the quantum anomaly in a manner similar to that described in our previous example, making use of the representation indices in Table 1 . The appropriate reductions are

$$
\begin{aligned}
\operatorname{tr} F^{2} & =\operatorname{tr} F_{a}^{2} \\
\operatorname{trace} F^{2} & =-2 \operatorname{tr} F_{a}^{2} \\
\operatorname{trace} F^{4} & =-3\left(\operatorname{tr} F_{a}\right)^{2}+16 \operatorname{tr} F_{a}^{4},
\end{aligned}
$$

where the subscript a now denotes $S O(16)$. Once again, the first of these should be substituted in the classical (inflow) anomaly, while the second two should be substituted in the quantum anomaly.

Using the three distinct cases which we have so far addressed, corresponding to the choices where $\beta$ breaks $E_{8}$ to $E_{8}, E_{7} \times S U(2)$ or $S O(16)$, we have enough data to completely determine an interesting set of solutions to our anomaly factorization problem. These solutions conform to our expectations by assembling into hierachies as described previously.

On a given $\alpha \beta$-plane, such as that depicted by the intersection point in Figure 1, the net six-dimensional anomaly $I_{8}$ is determined by adding up all six terms in (3) and (4). One then substitutes identities, such as (8) and (9), relevant to the particular $E_{8}$ breaking pattern being considered, in the manner explained above. What results is a polynomial 
with terms proportional to the each of $\operatorname{tr} R^{4},\left(\operatorname{tr} R^{2}\right)^{2}, \operatorname{tr} R^{2} \wedge \operatorname{tr} F^{2}, \operatorname{tr} R^{2} \wedge \operatorname{tr} F^{2},\left(\operatorname{tr} F^{2}\right)^{2}$, $\operatorname{tr} F^{2} \wedge \operatorname{tr} \mathcal{F}^{2}, \operatorname{tr} F^{4}$, and $\operatorname{tr} \mathcal{F}^{4}$, where $F$ stands generically for factors in the residual group $\mathcal{G} \subset E_{8}$ which survives the $\beta$ projection (we have denoted these $F_{a}$ and $F_{b}$ above) and $\mathcal{F}$ stands generically for factors in any gauge group associated with twisted matter.

Note that we have allowed for twisted fields which are either six or seven dimensional. In the former case, we refer to $N=1$ fields living exclusively on the $\alpha \beta$-plane under consideration. In the latter, we refer to vector adjoint supermultiplets living on the seven-dimensional $\beta$-plane which intersects this $\alpha \beta$-plane. The seven-dimensional fields will contribute to the anomaly with a tell-tale factor of two, as described above. Computationally, we accomodate both of these cases simultaneously by formally allowing $n_{T}$ tensors, $n_{V}$ vectors and $n_{H}$ hypermultiplets, where $n_{V}$ and $n_{H}$ can assume half-integral values. The (formal) appearance of half-integer numbers of multiplets then indicates that the associated matter is seven-dimensional.

Keeping the twisted matter arbitrary, we determine $I_{8}$ by adding up all six contributions in (3) and (4). For any choice of $\mathcal{G} \subset E_{8}$, we reduce the various $E_{8}$ traces to $\mathcal{G}$ traces according to the scheme described above. This process provides us with a provisional form of the local anomaly. It remains provisional since the twisted contribution remains to be resolved. Nevertheless, we can extract our first bits of useful information. Since anomaly cancellation is possible only if $I_{8}$ either vanishes identically or reduces to a sum of perfect squares, it follows that any nonfactorizable terms in $I_{8}$ must vanish. The vanishing of the $\operatorname{tr} R^{4}$ term requires

$$
n_{H}-n_{V}=30 g-29 n_{T}+\frac{1}{2 f}(244-h)+\frac{1}{f}\left(V_{B}-H_{B}\right) .
$$

This constraint is the local version of the global constraint $N_{H}-N_{V}+29 N_{T}=273$, where $N_{H}, N_{V}$ and $N_{T}$ are the total number of hyper, vector and tensor multiplets in the entire orbifold, including all twisted and untwisted contributions. The relationship between the local constraint (10) and the global version is described in [5]. Note that (10) is invariant when $g \rightarrow g+1, n_{T} \rightarrow n_{T}+1$, and $n_{H} \rightarrow n_{H}+1$, consistent with expectations described previously.

Henceforth, we concentrate on the $S^{1} / \mathbf{Z}_{2} \times T^{4} / \mathbf{Z}_{2}$ orbifold. In this case, we have $(f, h)=(16,4)$, as described above, so that (10) becomes

$$
n_{H}-n_{V}=30 g-29 n_{T}+\frac{15}{2}+\frac{1}{16}\left(V_{B}-H_{B}\right)
$$

Since the left-hand side must be either an integer or a half-integer, it follows that $\left(V_{B}-\right.$ $\left.H_{B}\right) / 16$ must be integer or half-integer as well, since in this case $g$ is quantized in quarter-integer units. Each of the three $E_{8}$ breaking patterns which we have addressed, 


\begin{tabular}{c|c}
$\mathcal{G}$ & $n_{H}-n_{V}$ \\
\hline$E_{8}$ & $30 g+23$ \\
$E_{7} \times S U(2)$ & $30 g+9$ \\
$S O(16)$ & $30 g+7$
\end{tabular}

Table 2: Constraints linking the local magnetic charge and the multiplicities of twisted hyper and vector multiplets when $n_{T}=0$ for three possible $E_{8}$ breaking patterns.

$\mathcal{G}=E_{8}, E_{7} \times S U(2)$ and $S O(16)$, corresponding to $\left(V_{B}, H_{B}\right)=(248,0),(136,112)$ and $(120,128)$ respectively, respect this constraint. We restrict our study to these three breaking patterns.

The sytematic analysis of local anomaly cancellation proceeds as outlined above. We first seek solutions with no twisted tensor multiplets, so that $n_{T}=0$. In this case, $I_{8}$ must vanish identically. We consider each of the three possible $E_{8}$ breaking patterns described above, using the relevant values of $V_{B}$ and $H_{B}$ in each case. For these three possibilities, when $n_{T}=0$ equation (11) reduces to the constraints indicated in Table 2. Thus, if there are no local twisted tensor multiplets, anomaly cancellation implies the indicated correlations between the local magnetic charge and the multiplicities of twisted hyper and vector multiplets. Note that, in each case, extra twisted matter is required since the indicated multiplicites can not be made to vanish with any properly quantized choice of $g$. The challenge in the case $n_{T}=0$ is not only to identify the multiplicities of twisted states, but also to identify the twisted gauge groups, the representation content of the twisted matter, as well as values of $g, \eta$ and $\rho$ which satisfy the restrictions in Table 2 (which ensures cancellation of the local tr $R^{4}$ anomaly). Furthermore, these choices must provide for complete cancellation of all other terms in the full polynomial $I_{8}$. Satisfying all of these requirements is a highly restrictive demand.

We first look for a "basic" solution where $\mathcal{G}=E_{8}$. In this case, we find a unique solution to all of our constraints. This solution requires $g=-3 / 4$ and $\tilde{\mathcal{G}}_{7}=S U(2)$, which is broken as $S U(2) \rightarrow U(1)$ on the $\alpha \beta$-plane. In this case, the three adjoint $S U(2)$ fields provide one six-dimensional vector multiplet and two hypermultiplets on the $\alpha \beta$ plane. There are no further twisted fields. Thus, the gauge structure on the $\alpha \beta$-plane is $E_{8} \times U(1)$, under which the twisted fields transform as follows 


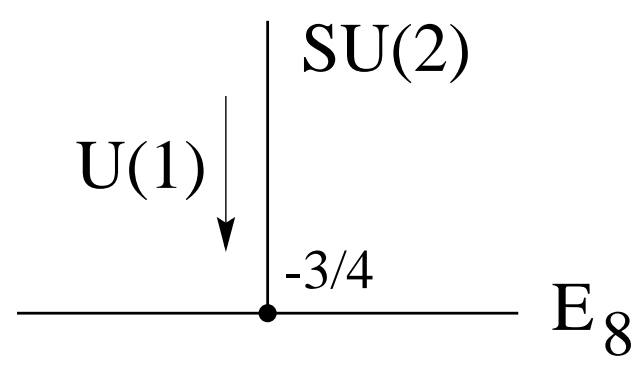

Figure 3: Vertex diagram corresponding to the "basic" solution in the $S^{1} / \mathbf{Z}_{2} \times T^{4} / \mathbf{Z}_{2}$ orbifold, as described in the text.

$$
\begin{array}{lcl}
\text { Vectors: } & \frac{1}{2} \mathbf{1}_{(\mathbf{0})} & n_{V}=1 / 2 \\
\text { Hypers: } & \frac{1}{2} \mathbf{1}_{(+\mathbf{1})} \oplus \frac{1}{2} \mathbf{1}_{(-\mathbf{1})} & n_{H}=1 / 2+1 / 2=1 \\
\text { Tensors: } & \text { None } & n_{T}=0,
\end{array}
$$

where the $U(1)$ charges are indicated in the parenthetical subscripts. This solution also requires $(\eta, \rho)=(1,0) \square$. In this case, the anomaly vanishes completely, as it should since there are no tensors to provide a local Green-Schwarz mechanism. The factor of one-half accompanying the twisted field representions indicate that these describe sevendimensional fields living on the $\beta$-plane, contributing via a chiral projection onto the embedded $\alpha \beta$-plane. We represent this local solution with the diagram shown in Figure 3. In Figure 3, the twisted gauge group $S U(2)$ is indicated next to the vertical line, signifying that this corresponds to $\tilde{\mathcal{G}}_{7}$. The magnetic charge associated with the intersection is indicated by the $-3 / 4$. There is no local $U(1)$ anomaly because the charges cancel, leaving a net $U(1)$ charge of zero.

Our basic solution has a two notable aspects. First, using the multiplicities listed above, we compute $n_{H}-n_{V}=1 / 2$, which is precisely the value specified in Table 2 for the case of unbroken $E_{8}$ and for the choice $g=-3 / 4$. The second notable aspect concerns the magnetic charge. For reasons described in [5], we attribute a topological significance to this number. Specifically, $g$ corresponds to an $E_{8}$ instanton number (associated with an instanton residing on the $\alpha \beta$-plane) minus the local contribution due to the nontrivial Euler character of the $K 3$ manifold. (We attribute the second of these to a local gravitational instanton, associated with a pointlike version of the ALE space needed to blow up the orbifold.) Since the Euler number of $K 3$ is 24, we divide this evenly over the 32 $\alpha \beta$-planes, so that the local gravitational contribution to $g$ should be exactly $-3 / 4$. Since

\footnotetext{
${ }^{12}$ The requirement that $\rho=0$ and the need for $U(1)$ gauge factors in the "basic" solution to $S^{1} / \mathbf{Z}_{2} \times$ $T^{4} / \mathbf{Z}_{2}$ orbifolds was also discussed in [6].
} 
we are considering the case of unbroken $E_{8}$, we assume there are no local gauge instantons. Therefore, the only contribution to $g$ should be the gravitational result of $-3 / 4$. It is gratifying that this number is required by our independent anomaly cancellation requirements.

When we impose $n_{T}=0$ and $I_{8}=0$ on the cases where $\beta$ breaks $E_{8}$ to $\mathcal{G}=E_{7} \times S U(2)$ or $\mathcal{G}=S O(16)$, we also find unique solutions with specialized values of $g$ and with specific twisted matter content. These solutions are described by the diagrams in the left-hand column of Figure 4. Note that, in each case, we require seven-dimensional $S U(2)$ vector multiplets. In the $\mathcal{G}=E_{7} \times S U(2)$ case, the seven-dimensional $S U(2)$ factor is identified with the $S U(2)$ factor in $\mathcal{G}$. This identification is indicated by the asterix on the two $S U(2)$ factors in the relevant diagram. As described previously, under these circumstances, $\alpha$ projects out the "vector" component of the seven-dimensional matter but preserves the "hyper" component. This is represented by the $H$ next to the arrow in the same diagram. In contrast to our "basic" solution, both of these new solutions involving local breakdown of $E_{8}$ require $(\eta, \rho)=(1,1)$. The nonvanishing of $\rho$ indicates that the $\beta$-planes support non-zero $S U(2)$ electric charge in these cases. The physics of this observation may prove interesting.

We interpret the $\beta$-induced $E_{8}$ symmetry breakdown as a reflection of instantons residing on the $\alpha \beta$-plane. Since there are two classes of gauge instantons which could reside on the $\alpha \beta$-planes, one contributing integer magnetic charge and the other contributing half-integer magnetic charge, we infer that solutions could exist with $g$ taking values at half-integer increments greater than the "basic" value of $-3 / 4$. This is precisely what we find; the $n_{T}=0$ solutions for $\mathcal{G}=E_{7} \times S U(2)$ and $\mathcal{G}=S O(16)$ require $g=-1 / 4$ and $g=+1 / 4$, corresponding to half-integer and integer valued instantons, respectively.

In the next phase of our systematic search for local anomaly-free vertices, we study the cases with $n_{T}=1$. In these cases, we impose that the anomaly factorizes as a complete square, $I_{8} \propto\left(Z_{4}\right)^{2}$, so that it can be cancelled by dynamics involving the self-dual tensor in the local twisted spectrum. Equation (11), which enforces the vanishing of the $\operatorname{tr} R^{4}$ term in $I_{8}$, is still important since tr $R^{4}$ cannot factorize. Once again, we consider each of the three $E_{8}$ breaking patterns discussed above, using the relevant values of $V_{B}$ and $H_{B}$ in each case. For these three possibilities, when $n_{T}=1$, equation (11) reduces to the constraints indicated in Table 3. Thus, if there is one local twisted tensor multiplets then local anomaly factorization implies the indicated correlations between the local magnetic charge and the multiplicities of twisted hyper and vector multiplets. Note that, in each case, extra twisted matter is still required since the indicated multiplicites can not be made to vanish with any properly quantized choice of $g$. The challenge, in the case 

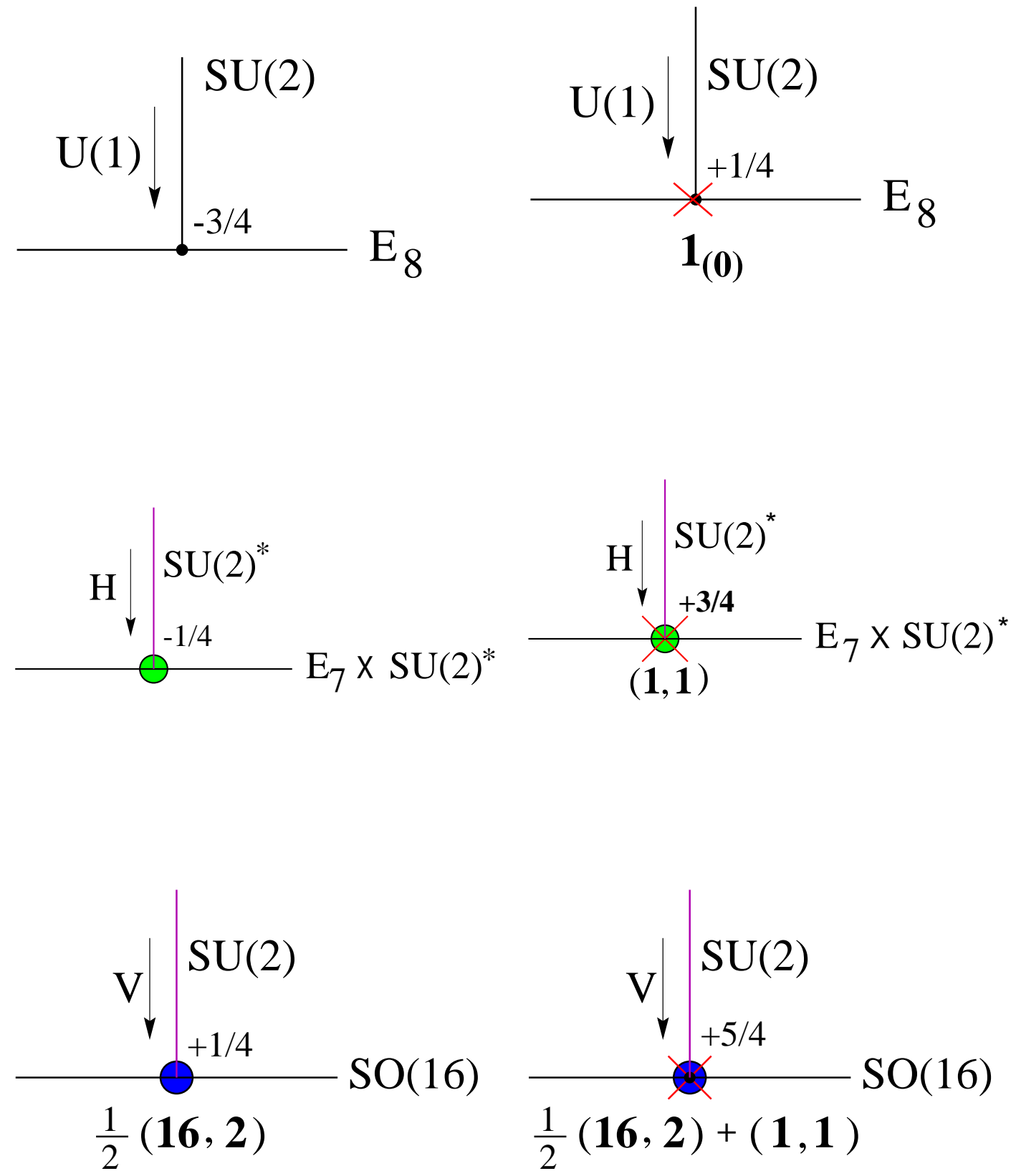

Figure 4: A collection of consistent orbifold vertices for the $S^{1} / \mathbf{Z}_{2} \times T^{4} / \mathbf{Z}_{2}$ orbifold for three possible $E_{8}$ breaking patterns and for the two possibilities $n_{T}=0$ and $n_{T}=1$. In the middle two diagrams, the asterix signifies that the indicated $S U(2)$ factors are identified. 


\begin{tabular}{c|c}
$\mathcal{G}$ & $n_{H}-n_{V}$ \\
\hline$E_{8}$ & $30 g-6$ \\
$E_{7} \times S U(2)$ & $30 g-20$ \\
$S O(16)$ & $30 g-22$
\end{tabular}

Table 3: Constraints linking the local magnetic charge and the multiplicities of twisted hyper and vector multiplets when $n_{T}=1$ for three possible $E_{8}$ breaking patterns.

$n_{T}=1$, is not only to identify multiplicities of twisted states, but also the twisted gauge groups, the represention content of the twisted fields, as well as values of $g, \eta$ and $\rho$ which can satisfy the restrictions in Table 3 (which ensures cancellation of the local $\operatorname{tr} R^{4}$ anomaly). Furthermore, they must provide the appropriate factorization of $I_{8}$. Satisfying these requirements is, again, a highly restrictive demand.

As before, we start with the case where $E_{8}$ remains unbroken. We again find a unique solution to our constraints, but this time with $g=+1 / 4$. Once again $\tilde{\mathcal{G}}_{7}=S U(2)$, and the seven dimensional gauge group is broken by $\beta$ on the $\alpha \beta$-plane as $S U(2) \rightarrow U(1)$. The only change to the twisted spectrum in the analogous $n_{T}=0$ solution is the addition of one singlet hypermultiplet to the local six-dimensional spectrum. Thus, the twisted fields transform under $E_{8} \times U(1)$ as follows

$$
\begin{array}{lcl}
\text { Vectors: } & \frac{1}{2} \mathbf{1}_{(\mathbf{0})} & n_{V}=1 / 2 \\
\text { Hypers: } & \frac{1}{2} \mathbf{1}_{(+\mathbf{1})} \oplus \frac{1}{2} \mathbf{1}_{(-\mathbf{1})} \oplus \mathbf{1}_{(0)} & n_{H}=1 / 2+1 / 2+1=2 \\
\text { Tensors: } & \mathbf{1}_{(0)} & n_{T}=0,
\end{array}
$$

where the $U(1)$ charges are again indicated in the parenthetical subscripts. This solution also requires $(\eta, \rho)=(1,0)$ The factors of one-half indicate that these fields describe a projection of a seven-dimensional multiplet, living on the $\beta$-fixed plane, via a chiral projection onto its boundary. This solution is shown in the upper right-hand diagram in Figure 4. In our diagrams, we indicate the presence of a twisted tensor multiplet by an $X$. In this case, the anomaly does not vanish, but is given by

$$
I_{8}=-\frac{1}{(2 \pi)^{3} 4} \frac{3}{16}\left(\operatorname{tr} R^{2}-2 \operatorname{tr} F^{2}\right)^{2} .
$$

Since this is proportional to a perfect square, it can be removed by a local Green-Schwarz mechanism mediated by the anti-self-dual tensor in the twisted tensor multiplet. 

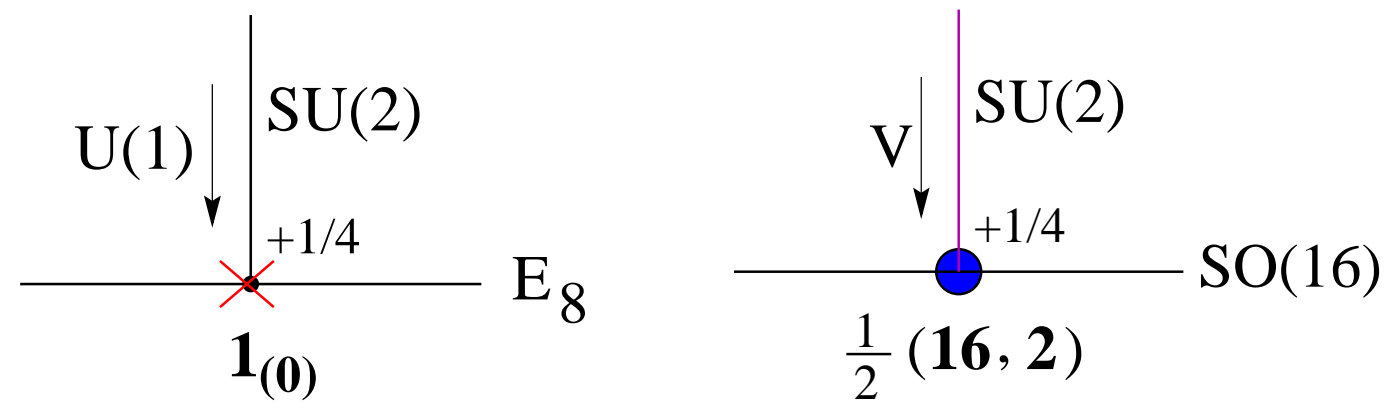

Figure 5: A two step process in which a fivebrane which has moved to a vertex smoothly deforms to an integrally-charged instanton.

When we impose $n_{T}=1$ and $I_{8} \propto\left(Z_{4}\right)^{2}$ on the cases where $\beta$ breaks $E_{8}$ to $\mathcal{G}=$ $E_{7} \times S U(2)$ or $\mathcal{G}=S O(16)$, we also find unique solutions with specialized values of $g$ and specific twisted matter content. The set of $n_{T}=1$ solutions corresponding to each of the our three choices for $\mathcal{G}$ are described by the diagrams in the right-hand column of Figure 4. In each of these cases, the presence of a twisted tensor multiplet is indicated by the $X$ on the relevant vertex. In these cases, the anomaly $I_{8}$ does not vanish but, rather, is given by a complete square. Therefore, the twisted tensor involves interesting dynamics. Notably, the cases where $E_{8}$ is broken require $\rho=1$. This is in contrast to the situation involving unbroken $E_{8}$, where $\rho=0$.

It is useful to compare the top right vertex-diagram in Figure 4 with the lower left vertex-diagram in that same figure. Since these two vertices have identical magnetic charge, we infer a transition whereby the fivebrane connects smoothly to an instanton, locally breaking $E_{8}$ to $S O(16)$. A subtle point concerns the electric charge of the associated seven-dimensional $\beta$-plane (the vertical line in these diagrams). In the unbroken $\left(E_{8}\right)$ phase we require $\rho=0$, so the seven-plane is not electrically charged. However, in the broken $\left(E_{7} \times S U(2)\right.$ or $\left.S O(16)\right)$ phases we require $\rho=1$. This process is shown by the two-diagram sequence depicted in Figure 5. 


\section{The Global Structure}

Now that we have tabulated some consistent local solutions, as classified in Figure 4, we can attempt to assemble these into a coherent global orbifold. There are extra constraints on this procedure, however, which need to be taken into account. The first of these is implied by the exactness of $d G$ and follows from integrating the Bianchi identity (2) over the five compact dimensions. Since this region has no boundary, the left-hand side of the integrated version of (2) vanishes due to Stokes theorem since the integrand is a total derivative. This implies that the net magnetic charge of the entire orbifold is zero. Without loss of generality, we can concentrate all of the magnetic sources either on the $\alpha \beta$-planes or on fivebrane worldvolumes. We therefore determine that

$$
N_{5}+\sum_{i=1}^{32} g_{i}=0,
$$

where $N_{5}$ is the number of fivebranes not residing on $\alpha \beta$-planes. Note that $N_{5}$ is necessarily a positive integer.

There is a unique "basic" global configuration satisfying this contraint for which neither of the $E_{8}$ factors is broken at any intersection. In this case, each $\alpha \beta$-plane carries a magnetic charge of $-3 / 4$. Since there are thirty-two $\alpha \beta$-planes, the proper magnetic balance is minimally achieved by including 24 fivebranes distributed randomly in the bulk of the orbifold. This situation is depicted by the first diagram in Figure 6 .

The diagrams in Figure 6 depict a portion of the orbifold in which only four of the thirty-two $\alpha \beta$-planes are shown. (The entire orbifold would be represented as in Figure 1.) One should think of these ladder-diagrams as assemblies of the individual vertex-diagrams represented in Figure 4. Thus, vertical lines represent seven-dimensional $\beta$-planes and horizontal lines represent ten-dimensional $\alpha$-planes. It is further understood that each of the two ten-planes supports local $E_{8}$ matter. The explicit factors of $E_{8}$ shown in the first diagram of Figure 6 indicate that the ten-dimensional $E_{8}$ matter is completely unbroken on each of the four $\alpha \beta$ intersections shown in that diagram. Similarly, any explicit group shown at a vertex in a ladder-diagram indicates the subgroup $\mathcal{G} \subset E_{8}$ which remains unbroken by $\beta$ at that indicated vertex. The $X$ 's indicate fivebranes. Each has a worldvolume which fills the six noncompact dimensions extending out of the plane of the diagram and carries unit magnetic charge.

The fivebranes are free to move about the diagrams. Notably, due to the consistent vertex indicated at the top of the right-hand column in Figure 4, the fivebranes are free to move to, and wrap, any of the vertices in the first diagram of Figure 6. Such a procedure is shown in the second diagram in Figure 6, where the arrow indicates that the fivebrane 

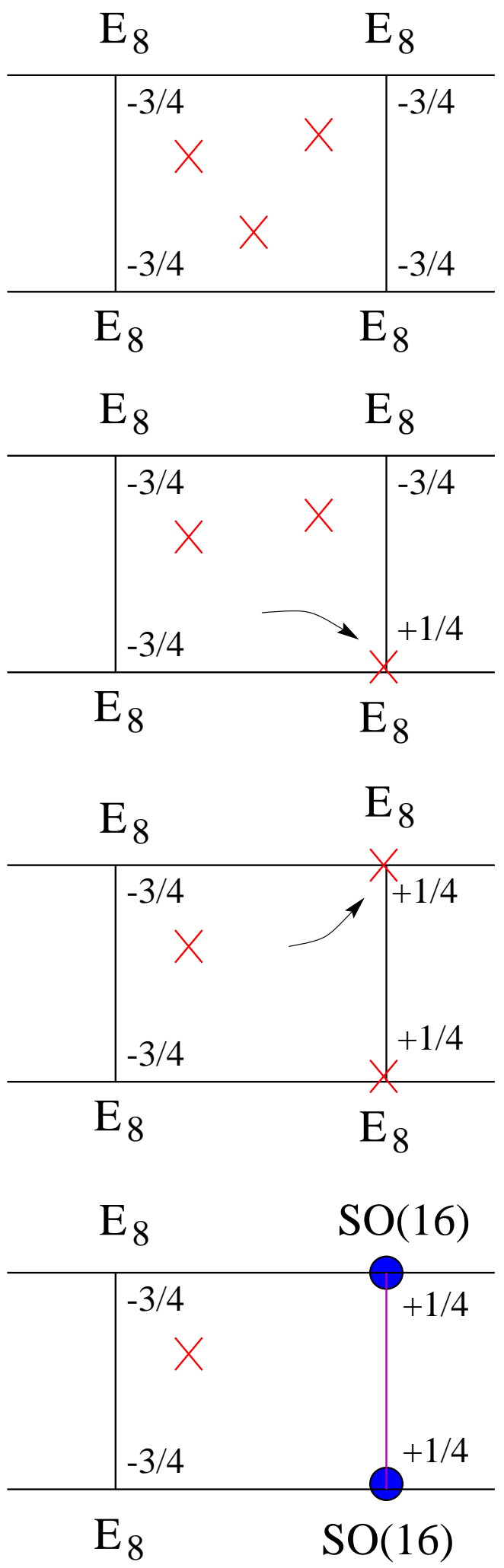

Figure 6: A global picture of a phase transition in the $S^{1} / \mathbf{Z}_{2} \times T^{4} / \mathbf{Z}_{2}$ orbifold mediated by fivebranes. 
has moved to, and wrapped, the indicated vertex. Note that, in this process, the local magnetic charge is increased by one unit, from $-3 / 4$ to $+1 / 4$. In addition, the twisted spectrum at that vertex is augmented by one tensor and one hypermultiplet. All of this is consistent with the absorption of a fivebrane. Transitions of this type can occur, without further constraints, at any vertex of a global orbifold configuration.

We would now like to consider the case where a fivebrane moves to a vertex and metamorphizes into a gauge instanton via a small instanton phase transition. As discussed above, this results in the breaking of $E_{8}$ to one of its subgroups. Previously in this paper, such transitions have been analyzed at a single vertex only. However, within the context of a global orbifold configuration, it is necessary to insure that such a phase transition is compatible with the structure of the surrounding vertices. This puts additional, rather strong, constraints on the allowed phase transitions. The pertinent issue involves the electric charge $\rho$ of the $\beta$-planes. Since the unbroken $\left(E_{8}\right)$ phases correspond to $\rho=0$ and the broken phases $\left(E_{7} \times S U(2)\right.$ or $\left.S O(16)\right)$ correspond to $\rho=1$, and since $\rho$ is associated with an entire seven-dimensional $\beta$-plane (i.e. an entire vertical line in one of our ladderdiagrams), it would seem that instanton transitions on vertices should only occur in pairs. According to this interpretation, the lone $n_{T}=1$ vertex shown in the second diagram in Figure 6 can smoothly connect to an instanton only if another fivebrane first moves to the complementary six-plane on the top of the ladder-diagram, as depicted in the third diagram in Figure 6. This enables each of the fivebranes to then smoothly connect to instantons, through processes of the sort shown in Figure 6, simultaneously turning on an electric charge $\rho=1$ on the interpolating seven-plane. Thus, the sequence depicted in Figure 5 describes a fivebrane-mediated transition which smoothly connects an $E_{8} \times E_{8}$ phase of the moduli space to an $S O(16) \times S O(16)$ phase.

It is puzzling that the value of the electric parameter $\rho$, which is ostensibly derived from a seven-dimensional Chern-Simons coupling, changes from zero to one when the described transitions take place. This is puzzling because if $\rho$ is a mere coupling constant, its value should not be subject to intermittent change. (On the other hand, we are able to justify a related change in the magnetic charge $g$, since this has an understandable topological origin, which allows us to resolve such a change in the manner described previously.) We suppose that this issue has an interesting resolution. This curiosity was independently noticed and commented on in [6]. For the time being we allow a situation-dependent $\rho$ as an allowed rule. We hope to discuss this issue further in a future paper.

It is less clear how the half-integer instantons can emerge via smooth transitions involving fivebranes. One picture which suggests itself, however, is the following. We can imagine a fivebrane moving to one of the ten-planes, which smoothly connects in 


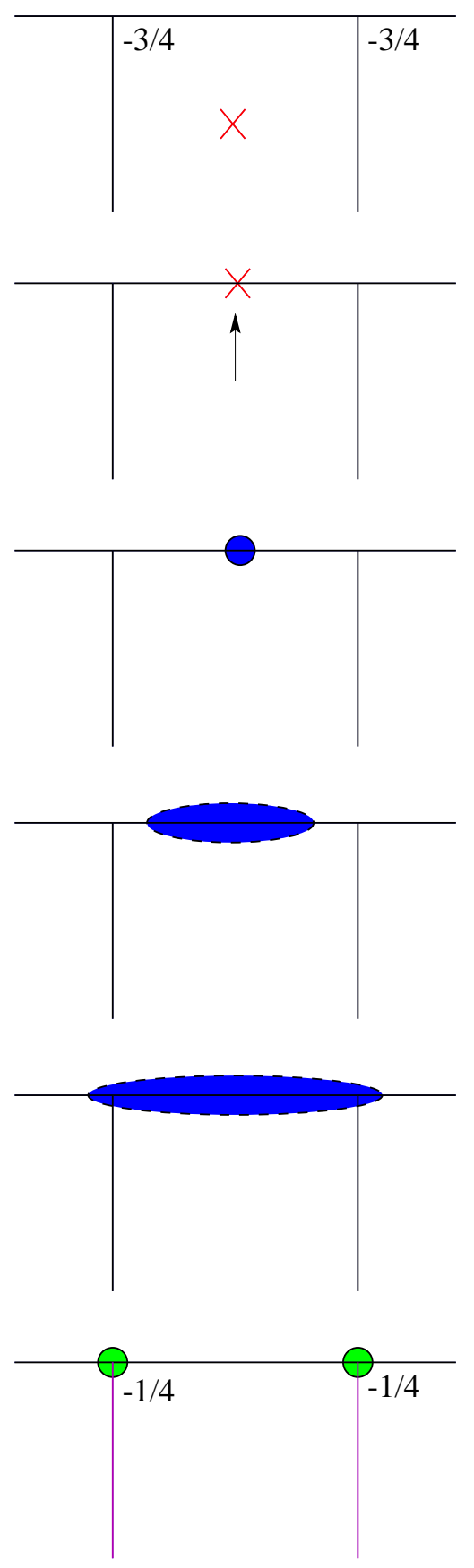

Figure 7: A fivebrane spawning a pair of half-integrally charged gauge instantons in a process involving an intermediate integrally charged instanton which grows to encompass two adjacent $\alpha \beta$-planes. 

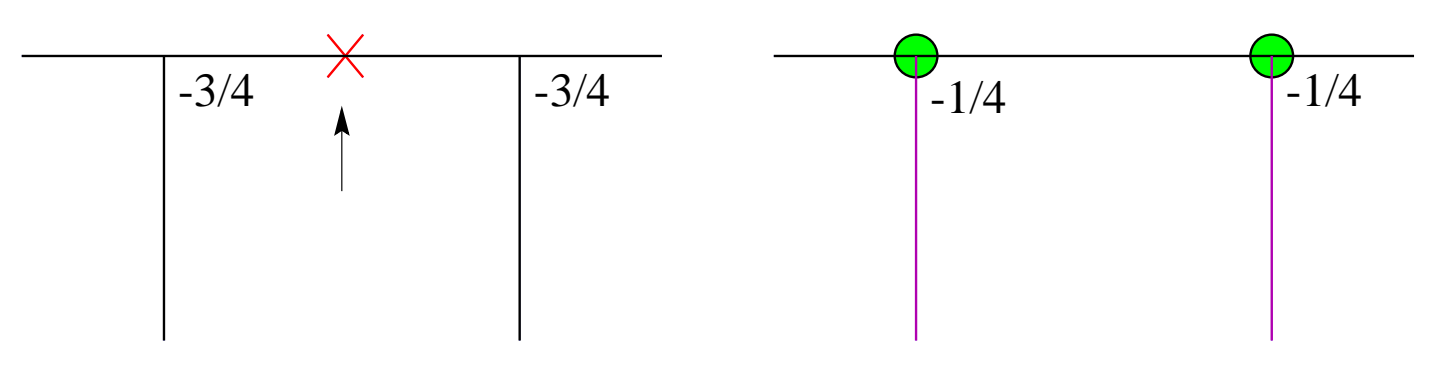

Figure 8: A two-step abbreviation of the process depicted in Figure 7. Heuristically, half of a fivebrane is wrapping each of the indicated vertices.

moduli space to a small ten-dimensional $E_{8}$ gauge instanton. Such a small instanton could then grow until it encompasses each of two fixed six-planes within the ten plane. We could imagine that this instanton then splits into two half-integer instantons, one localized on each of the two adjacent $\alpha \beta$-planes. This process is shown by a sequence of diagrams in Figure 7. We abbreviate this transition by the two-step sequence depicted in Figure 8. We can describe this process heuristically by saying that half of a fivebrane has wrapped each of the two involved $\alpha \beta$-planes. If such a thing were possible, then we could describe another phase transition as indicated by the sequence of diagrams in Figure 9. This depicts a fivebrane-mediated transition which smoothly connects an $E_{8} \times E_{8}$ phase of moduli space to an $S O(16) \times E_{7} \times S U(2)$ phase.

\section{Conclusions}

We have described some of the technology needed to resolve microscopic consistency issues in $M$-theory orbifolds. We have applied these techniques particularly to the $S^{1} / \mathbf{Z}_{2} \times T^{4} / \mathbf{Z}_{2}$ orbifold, and have presented an interesting set of consistent vertices, describing the twisted states residing on orbifold planes at intersecting points. By assembling consistent vertices we are able to build up consistent global orbifolds which describe different phases of moduli space. Our construction rather nicely indicates the possibility of phase transitions involving $M$-fivebranes as mediators. There remain intriguing conceptual issues which we intend to discuss and hope to resolve in forthcoming papers.

By resolving a larger set of consistent local vertices, thereby enlarging the number of diagrams in Figure 4, we should be able to significantly enrich our understanding of the possible allowed global configurations, and of the implied interconnectedness amongst phases in the moduli spaces associated with various orbifold compactifications indicated by the transitions which our diagrams suggest. It should prove interesting to apply our techniques to other orbifolds as well. 

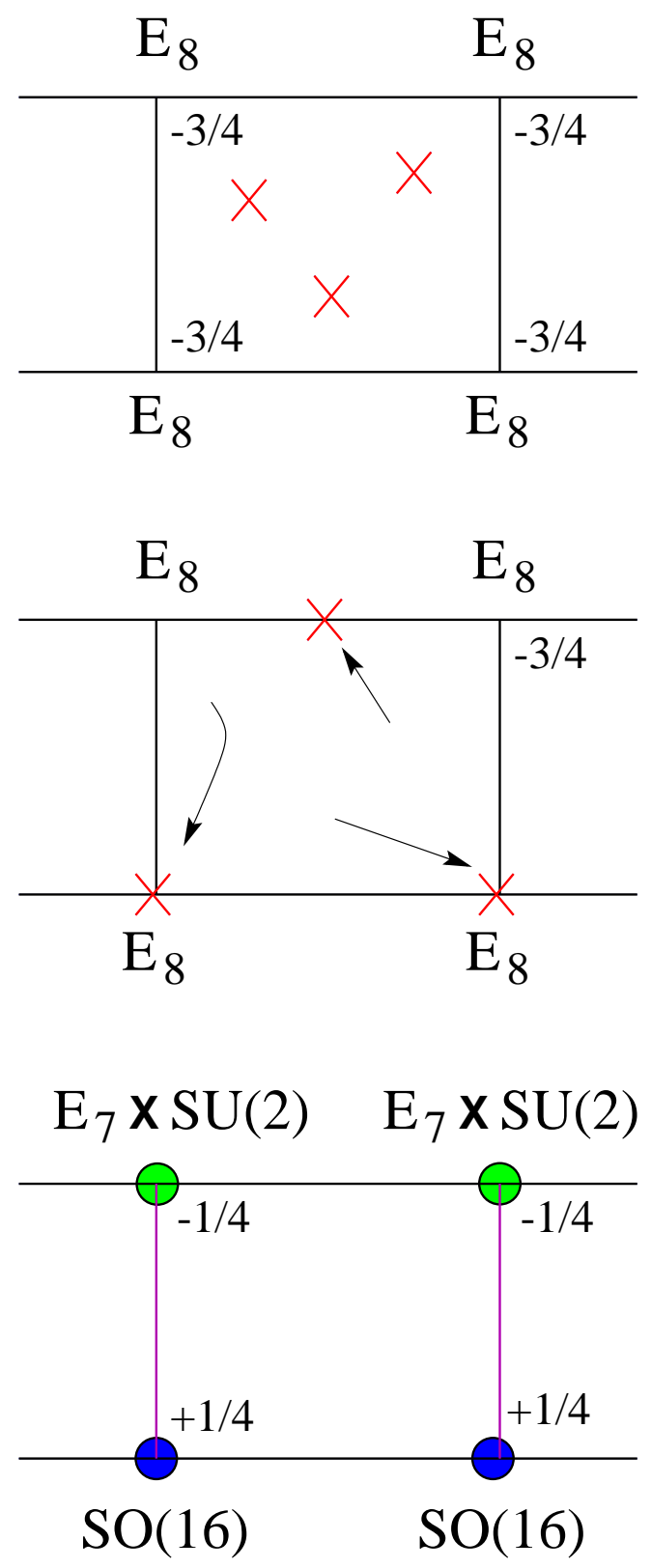

Figure 9: An global picture of a phase transition in the $S^{1} / \mathbf{Z}_{2} \times T^{4} / \mathbf{Z}_{2}$ orbifold, mediated by fivebranes. 


\section{Acknowledgements}

This work is supported by the European Commission TMR programme ERBFMRXCT96-0045, and the work of B.A.O. is supported by the Alexander von Humboldtfoundation. We would like to acknowledge helpful emails from Luis Alvarez-Gaume, Paul Aspinwall, Bernard de Wit and Bert Schellekens, and helpful comments by Vadim Kaplunovsky. In addition, we are grateful to Stefan Theisen for pointing out an error in the previous version of this paper pertaining to the pseudoreality of a hypermultiplet representation.

\section{References}

[1] P.Hořava and E.Witten, Heterotic and Type I String Dynamics from Eleven Dimensions, Nucl.Phys. B475 (1996) 94-114, hep-th/9510209.

[2] P.Hořava and E.Witten, Eleven-Dimensional Supergravity on a Manifold with Boundary, Nucl.Phys. B460 (1996) 506-524, hep-th/9603142.

[3] K.Dasgupta and S.Mukhi, Orbifolds of M-theory, Nucl.Phys. B465 (1996) 399-412, hep-th/9512196.

[4] E.Witten, Five-branes and M-Theory on an Orbifold, Nucl.Phys. B463 (1996) 383397 , hep-th/9512219.

[5] M.Faux, D.Lüst and B.A.Ovrut, Intersecting Orbifold Planes and Local Anomaly Cancellation in M-theory, Nucl.Phys. B554 (1999) 437-483, hep-th/9903028

[6] V. Kaplunovsky, J. Sonnenschein, S. Theisen, S. Yankielowicz, On the Duality between Perturbative Heterotic Orbifolds and $M$-Theory on $T^{4} / Z_{N}$, hep-th/9912144.

[7] K. Meissner and M. Olechowski, Anomaly Cancellation in M-theory on Orbifolds, hep-th/0003233.

[8] Adel Bilal, Jean-Pierre Derendinger, Roger Sauser, M-Theory on $S^{1} / Z_{2}$ : New Facts from a Careful Analysis, hep-th/9912150.

[9] E.Witten, On Flux Quantization in M-Theory and the Effective Action, J. Geom. Phys. 22 (1977) 1-13, hep-th/9609122. 
[10] A. Lukas, B. A. Ovrut, K. S. Stelle and D. Waldram, The Universe as a Domain Wall, Phys.Rev. D59 (1999) 086001; R. Donagi, A. Lukas, B. A. Ovrut and D. Waldram, Non-Perturbative Vacua and Particle Physics in M-Theory, JHEP 9905 (1999) 018; A. Lukas, B. A. Ovrut, K.S. Stelle and D. Waldram Cosmological Solutions of Horava-Witten Theory, Phys.Rev. D59 (1999) 086001; R. Donagi, B. A. Ovrut and D. Waldram, Moduli Spaces of Fivebranes on Elliptic Calabi-Yau Threefolds, JHEP 9911 (1999); M. Braendle, A. Lukas and B. A. Ovrut Heterotic M-Theory Cosmology in Four and Five Dimensions, hep-th/0003256.

[11] T. van Ritbergen, A.N. Schellekens, J.A.M. Vermaseren, Group theory factors for Feynman diagrams, Int.J.Mod.Phys. A14 (1999) 41-96, hep-ph/9802376.

[12] M.Green, J.A.Harvey and G.Moore, I-Brane Inflow and Anomalous Couplings on D-Branes, Class.Quant.Grav. 14 (1997) 47-52, hep-th/9605033

[13] B. A. Ovrut, T. Pantev and J. Park, Small Instanton Transitions in Heterotic MTheory, hep-th/0001133.

[14] E.Witten, Phase Transitions in M-theory and F-Theory, hep-th/9603150.

[15] E.Bergshoeff, E.Sezgin and A.van Proeyen, Superconformal Tensor Calculus and Matter Couplings in Six-Dimensions, Nucl.Phys. B264 (1986) 653-86.

[16] B.de Wit, P.G.Lauwers and A.van Proeyen, Lagrangians of $N=2$ SupergravityMatter Systems, Nucl.Phys. B255 (1985) 569-608.

[17] R.Slansky, it Group Theory for Unified Model Building, Phys.Rep. 79 (1981) 1-128. 